\title{
Carrier Multiplication in InAs Nanocrystal Quantum Dots with an Onset Defined by the Energy Conservation Limit
}

\author{
Richard D. Schaller, ${ }^{*}$ Jeffrey M. Pietryga, and Victor I. Klimov* \\ Chemistry Division, C-PCS, Los Alamos National Laboratory, \\ Los Alamos, New Mexico 87545
}

Received August 14, 2007; Revised Manuscript Received September 14, 2007

\begin{abstract}
Carrier multiplication (CM) is a process in which absorption of a single photon produces not just one but multiple electron-hole pairs (excitons). This effect is a potential enabler of next-generation, high-efficiency photovoltaic and photocatalytic systems. On the basis of energy conservation, the minimal photon energy required to activate $\mathrm{CM}$ is two energy gaps $\left(2 E_{\mathrm{g}}\right)$. Here, we analyze $\mathrm{CM}$ onsets for nanocrystal quantum dots (NQDs) based upon combined requirements imposed by optical selection rules and energy conservation and conclude that materials with a significant difference between electron and hole effective masses such as III-V semiconductors should exhibit a CM threshold near the apparent $2 E_{\mathrm{g}}$ limit. Further, we discuss the possibility of achieving sub-2 $E_{\mathrm{g}} \mathrm{CM}$ thresholds through strong exciton-exciton attraction, which is feasible in NQDs. We report experimental studies of exciton dynamics (Auger recombination, intraband relaxation, radiative recombination, multiexciton generation, and biexciton shift) in InAs NQDs and show that they exhibit a CM threshold near $2 E_{g}$.
\end{abstract}

Carrier Multiplication Thresholds in Bulk and Nanocrystalline Semiconductors. It has recently been established that carrier multiplication $(\mathrm{CM})$, the production of multiple excitons following absorption of a single photon of sufficient energy, is efficient within the range of solar photon energies in semiconductor nanocrystal quantum dots (NQDs). ${ }^{1-9}$ This finding is important because CM can potentially provide increased power conversion efficiency in low-cost, singlejunction photovoltaics via an enhanced photocurrent. ${ }^{10,11}$ To obtain optimum photovoltaic power conversion efficiency using a single semiconductor material with energy gap $E_{\mathrm{g}}$, it is desirable that the $\mathrm{CM}$ onset energy is minimal and that the CM efficiency above the onset is as large as is allowed by energy conservation.

According to energy conservation, the apparent minimal photon energy that is required to activate $\mathrm{CM}\left(\hbar \omega_{\mathrm{CM}}\right)$ is simply $2 E_{\mathrm{g}}$. However, the CM thresholds observed in bulk materials are significantly larger than this value. ${ }^{12,13}$ In bulk semiconductors, the generally accepted mechanism for multiexciton generation is impact ionization in which a highenergy electron (or a hole) transfers its energy to a valenceband electron that is excited across the energy gap. ${ }^{14}$ The CM threshold in this case is determined not only by conservation of energy but also by conservation of transla-

\footnotetext{
* Corresponding authors. E-mail: rdsx@lanl.gov (R.D.S.); klimov@ lanl.gov (V.I.K.).
}

tional momentum, which increases the threshold above $2 E_{\mathrm{g}}$. For example, using a free-particle approximation ${ }^{15,16}$ and applying both energy and momentum conservation laws, one can obtain that the CM threshold is approximately $4 E_{\mathrm{g}}$ (Figure 1a).

In NQDs, translational momentum conservation is relaxed. Therefore, one might expect that the CM threshold could reach the $2 E_{\mathrm{g}}$ limit as defined by energy conservation. However, available experimental data indicate that $\hbar \omega_{\mathrm{CM}}$ is still greater than twice the energy gap in such materials. For example, in $\mathrm{PbSe}$ and $\mathrm{PbS}$ NQDs, it is close to $3 E_{\mathrm{g}},{ }^{1,5,8}$ while it is $\sim 2.5 E_{\mathrm{g}}$ in CdSe NQDs. ${ }^{3}$ These observations can be understood by taking into account the fact that, in addition to energy conservation, the CM process in NQDs is also governed by selection rules that determine the magnitudes of the matrix elements of the electron-photon interaction and carrier-carrier Coulomb coupling.

Because the mechanism for CM in NQDs is still a subject of debate, the exact form of the selection rules also remains controversial. However, available experimental observations indicate that CM thresholds in the NQD systems studied so far can be understood in terms of a combination of optical selection rules that control the photon absorption event, and energy conservation, which governs promotion of the secondary electron across the energy gap (Figure 1b). These observations can be rationalized by using the following 

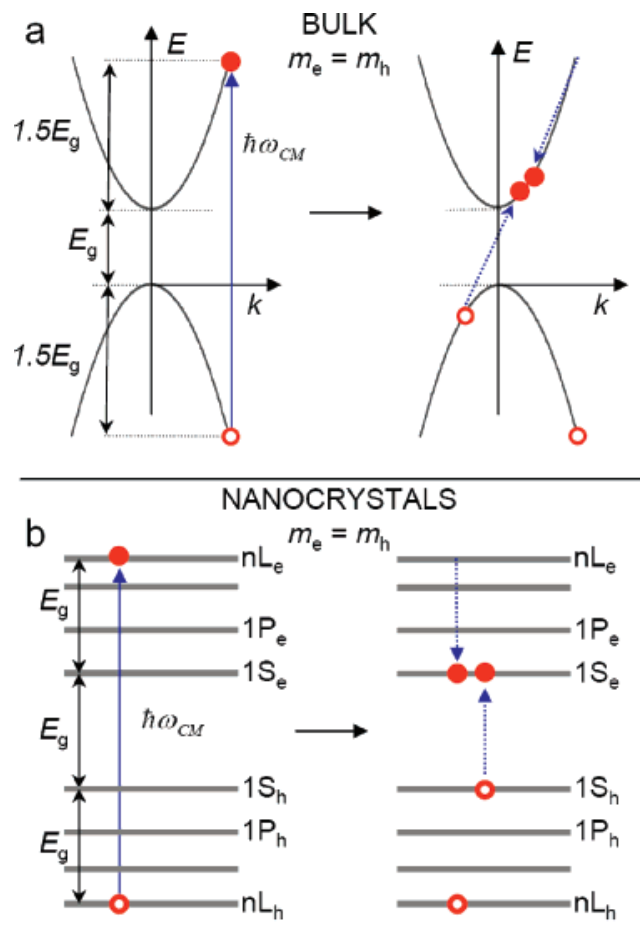

initial photoexcitation

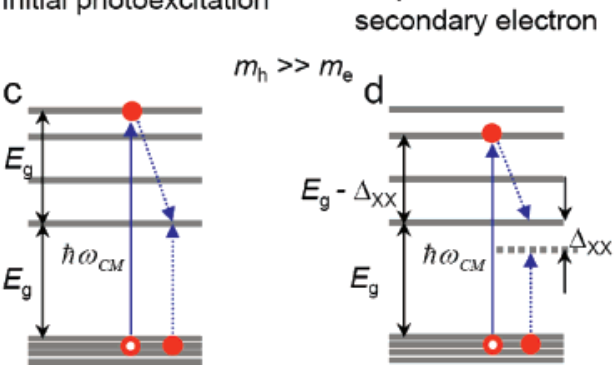

Figure 1. Carrier multiplication thresholds in bulk semiconductors and NQDs. (a) Because of restrictions imposed by energy and translational momentum conservation at both photoexcitation (left) and impact-ionization (right) steps, the CM threshold in bulk materials is greater than $2 E_{\mathrm{g}}$. In the case of semiconductors with identical electron and hole masses $\left(m_{\mathrm{e}}=m_{\mathrm{h}}\right)$, the conservation laws result in the $4 E_{\mathrm{g}} \mathrm{CM}$ threshold. (b) In NQDs, the initial photoexcitation step (left) is controlled by optical-selection rules according to which the energy of a photon in excess of the energy gap is distributed between the electron and the hole inversely proportionally to their effective masses. Because of the zero-dimensional character of the NQD density of states, one can disregard translational momentum conservation at the secondary-electron generation step and only account for energy conservation. Elimination of restrictions associated with momentum conservation reduces the $\mathrm{CM}$ threshold, which in the case of $m_{\mathrm{e}}=m_{\mathrm{h}}$ becomes equal to $3 E_{\mathrm{g}}$. (c) $\mathrm{CM}$ onset can approach $2 E_{\mathrm{g}}$ if $m_{\mathrm{e}} \ll m_{\mathrm{h}}$ and, hence, most of the energy deposited by a photon is acquired by the electron. (d) Coulomb X-X interaction energy, $\Delta_{\mathrm{XX}}$, can reduce the energy required to generate an additional exciton in the presence of an existing exciton and, hence, lower the $\mathrm{CM}$ onset below the apparent limit of $2 E_{\mathrm{g}}$.

considerations. Within a simple two-band model, ${ }^{17}$ optical excitation of a NQD preserves the symmetry of the envelope wave function and, hence, both a photoexcited electron and a hole are characterized by the same set of quantum numbers that determine the angular momentum $(L)$ and the number of nodes in its radial component $(n)$. As a result, the energies of photoexcited electrons $\left(E_{\mathrm{e}}\right)$ and holes $\left(E_{\mathrm{h}}\right)$ are given by
$E_{\mathrm{e}, \mathrm{h}}=\left(\hbar^{2} \phi_{n L}^{2} / 2 m_{\mathrm{e}, \mathrm{h}} R^{2}\right)$, where $m_{\mathrm{e}}$ and $m_{\mathrm{h}}$ are electron and hole effective masses, $R$ is the NQD radius, and $\phi_{n L}$ is the $n$th root of the Bessel function of the $L$ th order. This expression indicates that the energy of a photon in excess of the energy gap, $\left(\hbar \omega-E_{\mathrm{g}}\right)$, is distributed between the electron and the hole in inverse proportion to their effective masses: $E_{\mathrm{e}} / E_{\mathrm{h}}=m_{\mathrm{h}} / m_{\mathrm{e}}$.

Next we apply energy conservation, according to which promotion of the secondary electron across the energy gap can only occur if the greater of two energies $E_{\mathrm{e}}$ and $E_{\mathrm{h}}$ is equal to $E_{\mathrm{g}}\left[\max \left(E_{\mathrm{e}}, E_{\mathrm{h}}\right)=E_{\mathrm{g}}\right]$, which leads to the following expression for $\hbar \omega_{\mathrm{CM}}$ (assuming that $m_{\mathrm{e}} \leq m_{\mathrm{h}}$ ): $\hbar \omega_{\mathrm{CM}}=$ $E_{\mathrm{g}}\left(2+m_{\mathrm{e}} / m_{\mathrm{h}}\right)$, as introduced by us in ref 3 . In the case of $m_{\mathrm{e}}=m_{\mathrm{h}}$, it predicts a $3 E_{\mathrm{g}}$ threshold. This value is smaller than that obtained for bulk semiconductors $\left(4 E_{\mathrm{g}}\right)$ for $m_{\mathrm{e}}=$ $m_{\mathrm{h}}$, which is a direct consequence of the fact that for NQDs we disregard translational momentum conservation at the secondary-electron excitation step. Of course, simple twoband models forego the complexity of the detailed electronic structure of a nanocrystalline material associated, for example, with multi-sub-band character of the valence band in II-VI and III-V semiconductors. However, such twoband models should be a good guide for predicting the energetic onset of CM. This is because the lowest-energy electronic transition that can possibly result in an electron with a sufficiently high energy to undergo CM likely originates from the valence sub-band with the largest carrier mass (e.g., the heavy-hole sub-band in InAs).

The expression derived above agrees reasonably well with available experimental results. In lead salt NQDs, electron and hole masses are nearly identical, therefore, the expected $\mathrm{CM}$ threshold is $3 E_{\mathrm{g}}$. This value is in good agreement with $\mathrm{CM}$ thresholds measured for both PbSe and PbS NQDs. ${ }^{1,5}$ In $\mathrm{CdSe}$, the electron is approximately six times lighter than the hole and, therefore, $\hbar \omega_{\mathrm{CM}}$ is expected to drop to $\sim 2.2 E_{\mathrm{g}}$. Indeed, the measured $\mathrm{CM}$ threshold for CdSe NQDs $\left(2.5 E_{\mathrm{g}}\right)$ is lower than those in PbSe and PbS NQDs. Some discrepancy between estimated and measured values of $\hbar \omega_{\mathrm{CM}}$ can be attributed to significant anisotropy of the valence band in CdSe and the presence of multiple valence sub-bands.

On the basis of the considerations described above, one would expect that materials with a significant difference between $m_{\mathrm{e}}$ and $m_{\mathrm{h}}$ should approach the $2 E_{\mathrm{g}}$ limit for the $\mathrm{CM}$ activation threshold (see Figure 1c). Potential candidates for having reduced $\mathrm{CM}$ thresholds are the III-V semiconductors. For example, in InAs, $m_{\mathrm{e}} / m_{\mathrm{h}}$ is ca. 0.05 and this ratio is even smaller $(\sim 0.03)$ for InSb. Additionally, III-V NQDs of compositions such as InAs and InSb have an energy gap that is tunable through the near-infrared, which is ideally suited for optimizing the performance of NQD-based photovoltaic structures. ${ }^{10}$ Finally, the use of III-V nanostructures can help to solve issues of material toxicity, which is a concern in the case of NQDs based on lead salts.

III-V NQDs are also well-suited for studies of the effect of Coulomb exciton-exciton $(\mathrm{X}-\mathrm{X})$ interactions on the $\mathrm{CM}$ activation thresholds. The dielectric constants of III-V semiconductors are much smaller than those of lead salts. 
Therefore, $\mathrm{X}-\mathrm{X}$ interaction energy $\left(\Delta_{\mathrm{XX}}\right)$ is expected to be greater in InAs NQDs compared to that of, e.g., PbSe NQDs. An interesting aspect of the studies of $\mathrm{X}-\mathrm{X}$ Coulomb coupling in the context of $\mathrm{CM}$ is the possibility of reducing the $\mathrm{CM}$ threshold below the apparent minimum of $2 E_{\mathrm{g}}$. Because of the strong $\mathrm{X}-\mathrm{X}$ attraction $\left(\Delta_{\mathrm{XX}}<0\right)$ that exists in NQDs, ${ }^{18}$ the energy of a NQD two-exciton state (biexciton) can be significantly smaller than twice the single-exciton energy, resulting in a large biexciton binding energy $\left(\delta E_{\mathrm{XX}}\right)$, which is $\delta E_{\mathrm{XX}}=-\Delta_{\mathrm{XX}}$. For example, previous studies of CdSe NQDs indicate that the $\mathrm{X}-\mathrm{X}$ interaction energy can be as large as tens of meV. ${ }^{18}$ In the case of infrared materials, such values could represent a significant fraction of the NQD energy gap and, therefore, could appreciably lower the CM threshold. In materials with a significant disparity between electron and hole effective masses, the $\mathrm{X}-\mathrm{X}$ interactions can potentially shift the $\mathrm{CM}$ threshold even below $2 E_{\mathrm{g}}$ to values determined by the condition $\hbar \omega_{\mathrm{CM}}=2 E_{\mathrm{g}}-\left|\Delta_{\mathrm{XX}}\right|$ (see Figure 1d).

After we began measurements of CM in III-V NQDs, an initial study of $\mathrm{CM}$ in an InAs-CdSe-ZnSe core-shellshell NQD samples was reported in which transient conductivity and quasicontinuous-wave photoluminescence (PL) methods were used to detect CM. ${ }^{9}$ A promising result of this work was the observation of a high CM efficiency (1.6 exciton per absorbed photon) for a photon energy of $2.74 E_{\mathrm{g}}$, which is suggestive of a $2 E_{\mathrm{g}}$ limit. This previous study, however, did not determine either the CM energy onset or the spectral dependence of CM efficiency above the threshold, while both of those are important characteristics of the CM process.

Here, we study CM in InAs NQDs using a transientabsorption (TA) method originally introduced by us in ref 1. We first determine size-dependent rates of Auger recombination using direct excitation of multiexcitons at high pump fluences. The biexciton lifetimes increase rapidly with NQD size, as has been previously observed for other types of NQDs. ${ }^{19}$ With an understanding of the Auger recombination times, we then perform studies of CM efficiency as a function of excitation photon energy. We find that $\mathrm{CM}$ is efficient in InAs NQDs, with a measured quantum efficiency (QE) of photon-to-exciton conversion of up to $200 \%$, which corresponds to complete saturation of the lowest-energy, 2-fold degenerate $1 \mathrm{~S}$ electron level. We also directly measure the CM energy onset and observe that, for certain NQD sizes, it is near the energy-conservation-defined minimum of $2 E_{\mathrm{g}}$. The threshold for CM appears to exhibit some size dependence in this class of materials, which has not been observed previously for other types of NQDs studied in the context of CM. The growth of $\mathrm{QE}$ above the $\mathrm{CM}$ threshold is approximately linear with energy and is characterized by a slope (differential QE; ref 3) of $\sim 35 \% / E_{\mathrm{g}}$. We also examine a potential contribution of the $\mathrm{X}-\mathrm{X}$ interaction energy to the CM onset energy by analyzing the amplitude of a TA feature associated with the Coulomb-interaction-induced shift of the band-edge optical transition.

Experimental Methods. Colloidal InAs NQDs of controlled size were synthesized by following the report by
Guzelian et al. ${ }^{20}$ and were in some instances over-coated with successive layers of CdSe by using the method described by $\mathrm{Cao}$ et al. ${ }^{21}$ until the emission quantum yield was maximized (2-3 monolayers of CdSe). Dynamical studies were performed using a femtosecond (50 fs pulse width) TA system that was made tunable with two optical parametric amplifiers. Measurements were performed at room temperature on NQD samples dissolved in hexane or in the form of solid films (the form of the sample does not alter the processes of Auger recombination or CM). Pump pulses of controlled intensity, photon energy, and temporal delay were spatially overlapped with probe pulses that were tuned to the $1 \mathrm{~S}$ absorption feature of an NQD sample. In all TA measurements, we determine the average number of pump pulse photons absorbed per NQD, $N_{\text {eh }}$, that we can both calculate and experimentally verify. ${ }^{22,23}$ Time-resolved emission measurements were performed using an InP/InGaAs photomultiplier tube and photon-counting electronics.

To detect and quantify CM, we exploit distinct differences in population dynamics of single excitons versus multiexcitons in NQDs. In high-quality NQDs, single excitons decay on a slow (tens to hundreds of nanoseconds) time scale that is typically controlled by radiative recombination. Multiexcitons, in contrast, relax via efficient Auger recombination, which occurs on a fast (tens to hundreds of picosecond) time scale in all colloidal NQD materials reported to date ${ }^{1,24,25}$ and leaves a single exciton at a later time. TA dynamics monitored at the energy of the $1 \mathrm{~S}$ band-edge feature are dominated by state filling and exhibit a bleach amplitude that is primarily determined by the number of electrons in the $1 \mathrm{~S}$ state. The contribution from holes to the $1 \mathrm{~S}$ bleach is insignificant because of close separation between valenceband states, which results in small occupation numbers of individual hole levels. ${ }^{26,27}$

The pump-induced absorption change at the position of the $1 \mathrm{~S}$ transition, $\Delta \alpha_{1 S}$, provides a measure of the total number of carriers occupying the $1 \mathrm{~S}$ state averaged across the NQD ensemble. Because Auger recombination is nearly $100 \%$ efficient in materials that exhibit slow radiative recombination, CM quantum efficiency can be measured simply as the ratio of early time bleach amplitude divided by the amplitude of a long-lived "single-excitonic" background. In the case when multiexcitons are generated at high pump fluences via a traditional mechanism of sequential absorption of multiple photons, it is important to account for nonlinearity of the 1S TA response as a function of the average number of excitons per NQD. ${ }^{23}$ However, when multiexcitons are produced via $\mathrm{CM}$, the nonlinearity is insignificant if the average exciton multiplicity (defined as $\mathrm{QE} / 100 \%$ ) does not exceed the degeneracy of the $1 \mathrm{~S}$ level. ${ }^{6}$ The latter was the case in the present experiments, in which the measured QEs did not exceed 200\%.

Single-Exciton and Biexciton Dynamics. To determine $\mathrm{CM}$ efficiencies in III-V NQDs, we first characterize singleand multiexciton dynamics in these materials. Typical absorption and PL spectra of InAs core-only NQDs (diameter $=4.3 \mathrm{~nm}$, trioctylphosphine-capped) and InAs $-\mathrm{CdSe}$ core shell NQDs, which were prepared by following the reports 

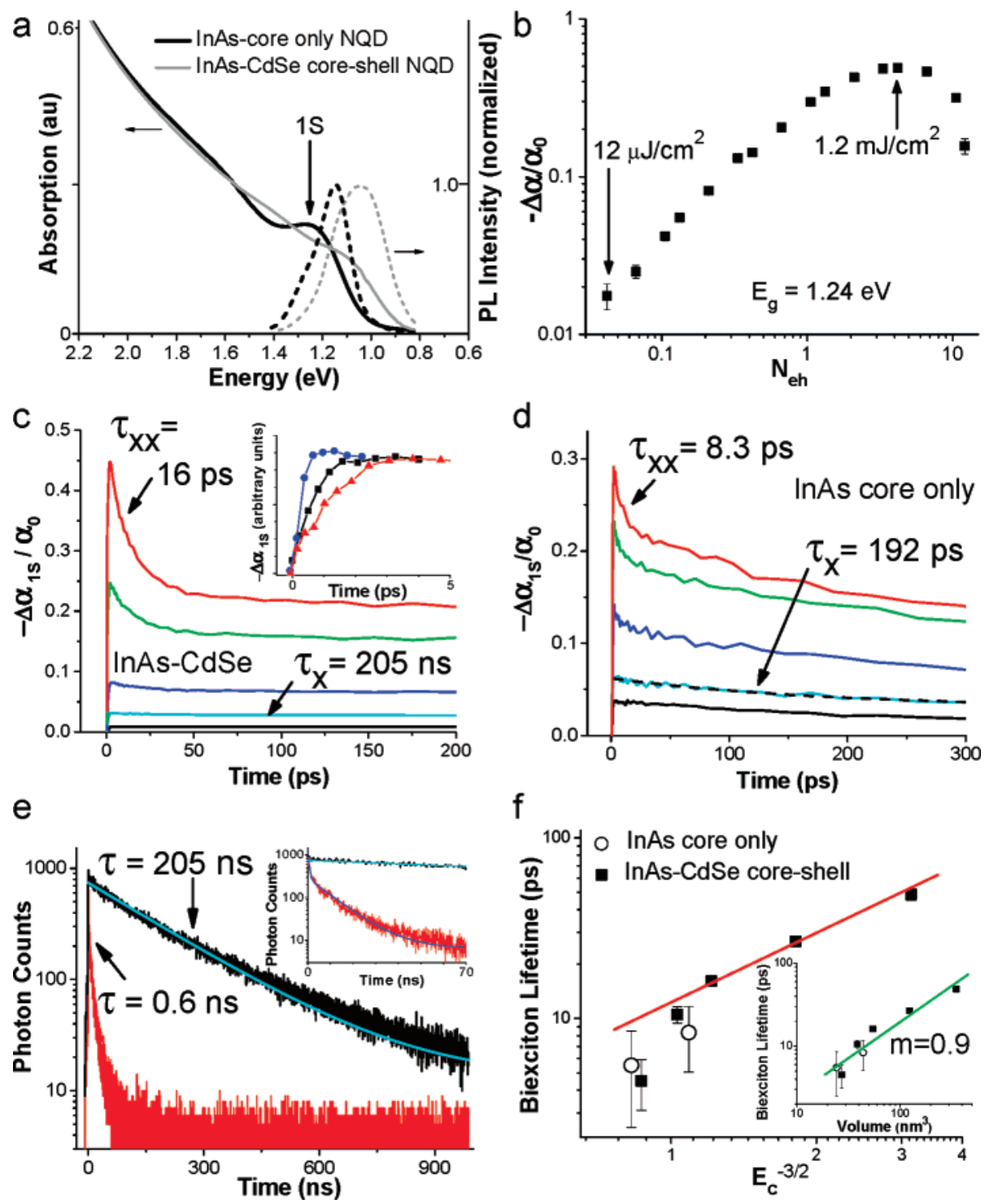

f

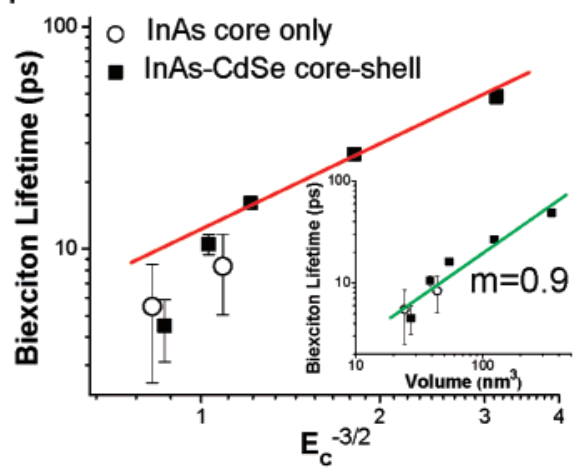

Figure 2. Single and multiexciton dynamics in InAs core and InAs-CdSe core-shell NQDs. (a) Absorption (solid lines) and emission (dashed lines) spectra of $4.3 \mathrm{~nm}$ diameter InAs core-only NQDs (black) and the same InAs core coated with a CdSe shell (gray). Distinct band edge absorption features are observed for the NQDs before and after CdSe shell growth and the Stokes shift for these samples remains constant, indicating type-I band alignment. (b) Pump intensity dependence of the transient absorbance $(\Delta \alpha)$ divided by the ground-state absorbance $\left(\alpha_{0}\right)$ measured at the position of the $1 \mathrm{~S}$ absorption maximum (1.24 eV for this InAs-CdSe core-shell NQD sample) with a temporal delay of $1.5 \mathrm{ps}$ after a $1.55 \mathrm{eV}$ excitation pulse. (c) Pump-intensity-dependent TA dynamics (1.55 eV pump photon energy) measured for an InAs-CdSe core-shell NQD sample (monitored at $1.24 \mathrm{eV}$ ) exhibit slow single-exciton relaxation dynamics at low pump intensity $\left(N_{\mathrm{eh}} \ll 1\right.$, black and cyan lines) and a faster relaxation component for higher pump intensity $\left(N_{\mathrm{eh}}>1\right.$, blue, green, and red lines). Rise times of the $1 \mathrm{~S}$ exciton bleach (inset) for three different InAs-CdSe NQD 1S exciton energies indicate rates of intraband relaxation decrease for lower $1 \mathrm{~S}$ exciton energy (corresponding to larger NQD size) (blue circles: $1 \mathrm{~S}=1.34 \mathrm{eV}, \tau_{\mathrm{r}}=0.35 \mathrm{ps}$; black squares: $1 \mathrm{~S}=$ $1.24 \mathrm{eV}, \tau_{\mathrm{r}}=0.66 \mathrm{ps}$; red triangles: $1 \mathrm{~S}=0.83 \mathrm{eV}, \tau_{\mathrm{r}}=1.21 \mathrm{ps}$ ). (d) Pump-intensity-dependent TA dynamics measured on an InAs core-only NQD sample (monitored at $1.30 \mathrm{eV}$ ). Here, single-exciton dynamics exhibit a decay constant of $\sim 200$ ps (fit based on measurements to $1.2 \mathrm{~ns}$ that are not shown), indicated by the dashed black line. (e) Time-resolved near-infrared PL measurements of InAs core-only (red trace) and InAs-CdSe core-shell NQDs (black trace) excited at $1.55 \mathrm{eV}$ and $N_{\text {eh }}<0.1$ show remarkable differences in single-exciton excited-state lifetime. Relaxation in the core-only material is clearly biexponential, with a fast time constant of $\sim 600$ ps (the width of the instrument response function is $\sim 400 \mathrm{ps}$ ), and a smaller amplitude component, with a time constant of $\sim 10.8 \mathrm{~ns}$. The core-shell material exhibits single-exponential relaxation with a much longer time constant of $205 \mathrm{~ns}$ (cyan line). Inset: early time dynamics for the same samples (blue line is a biexponential fit to the core-only NQD sample). (f) Biexciton lifetimes extracted from pump-intensity-dependent TA dynamics show an approximately linear change with $E_{\mathrm{c}}^{-3 / 2}$, which is roughly proportional to the NQD volume. Inset: comparison of biexciton lifetime with a NQD volume that is calculated based upon the InAs NQD sizes reported in ref 20.

of Guzelian et al. ${ }^{20}$ and Cao et al., ${ }^{21}$ respectively, are shown in Figure 2a. As reported by Cao et al., ${ }^{21}$ the lowest-energy absorption feature (referred to below as the $1 \mathrm{~S}$ exciton feature) red-shifts significantly upon growth of the shell and the emission quantum yield increases dramatically (by a factor of $\sim 100$ ). Also, the Stokes shift between the $1 \mathrm{~S}$ absorption feature and the PL band remains approximately constant upon growth of the CdSe shell, which indicates that type-I band alignment is maintained between the InAs core and the CdSe shell. ${ }^{21}$ 
Figure $2 \mathrm{~b}$ shows pump-intensity-dependent TA measurements performed on InAs-CdSe core-shell NQDs (here InAs core diameter is $3.9 \mathrm{~nm}$ ) using a probe pulse tuned to the energy of the $1 \mathrm{~S}$ absorption maximum $(1.24 \mathrm{eV})$ at a delay of $1.5 \mathrm{ps}$ after a $1.55-\mathrm{eV}$ excitation pulse of controlled intensity. The transient absorbance $(-\Delta \alpha)$ divided by the ground-state absorbance $\left(\alpha_{0}\right)$ exhibits initial linear growth with increasing pump intensity until the calculated number of photogenerated electron-hole pairs (excitons), $N_{\text {eh }}$, begins to exceed 1 (the absorption cross section is calculated ${ }^{22,23}$ to be $\left.\sigma_{1.55} \mathrm{ev}\left(\mathrm{cm}^{2}\right)=8.9 \times 10^{-17}[r(\mathrm{~nm})]^{3}\right)$. For $N_{\mathrm{eh}}>1$, $-\Delta \alpha / \alpha_{0}$ begins to saturate at a value of $\sim 0.5$. Saturation is typical of other NQD materials and is attributed to filling of the $1 \mathrm{~S}$ electron state, ${ }^{23,25,28}$ which is characterized by 2-fold degeneracy for InAs NQDs. The saturation value of $\left|\Delta \alpha / \alpha_{0}\right|$ is theoretically expected to approach 1 , but often does not, possibly as a result of photoinduced absorption. ${ }^{28,29}$ While changing the absolute value of $\Delta \alpha$, the potential contribution from photoinduced absorption is not expected to significantly affect $\mathrm{CM}$ efficiency measurements because they rely not on absolute but on relative values of TA signals (specifically, the ratio of the short- to long-time TA amplitudes).

Dynamics measured at the $1 \mathrm{~S}$ exciton absorption peak for several pump pulse intensities (using $1.55 \mathrm{eV}$ pump photon energy) are shown in Figure 2c. It can be seen in the inset of Figure $2 \mathrm{c}$ that the rise of the $1 \mathrm{~S}$ transient bleach occurs on a subpicosecond time scale (build-up time $\tau_{\mathrm{r}}=0.66 \pm$ $0.06 \mathrm{ps}$ for this sample; black squares). The $1 \mathrm{~S}$ rise time is also found to be dependent upon the $1 \mathrm{~S}$ exciton energy and becomes faster for higher exciton energy, which corresponds to smaller NQD size. Because the band-edge TA signal buildup is controlled by electron relaxation into to the lowestenergy $1 \mathrm{~S}$ state, a decrease of $\tau_{\mathrm{r}}$ for smaller NQD sizes indicates confinement-induced enhancement in electron intraband relaxation. This effect has been previously observed for, e.g., $\mathrm{CdSe}^{30}$ and $\mathrm{InP}^{31} \mathrm{NQDs}$ and has been attributed to high-efficiency, Auger-type electron-hole energy transfer. $^{32,33}$ The cooling rate that we measure for InAs NQDs (e.g., $\sim 0.5 \mathrm{eV} / \mathrm{ps}$ for NQDs with a $1 \mathrm{~S}$ absorption maximum at $1.24 \mathrm{eV}$ ) is consistent with rates observed for this process for CdSe NQDs. ${ }^{26}$

At low $1.55 \mathrm{eV}$ pump intensity, for which less than one exciton is generated per NQD, the $1 \mathrm{~S}$-exciton TA relaxation dynamics of the InAs-CdSe core-shell NQDs are very slow, with no discernible decay over the experimental time window. As the pump intensity is increased, a fast relaxation component (with a relaxation time on the order of tens of picoseconds) grows into the dynamics. TA studies performed on InAs core-only NQDs (probed at $1.30 \mathrm{eV}$ ) exhibit similar pump-power-dependent behavior except that the core-only material exhibits a single exciton decay time constant of 192 \pm 22 ps (Figure 2d), similar to the report of Chen et al. ${ }^{34}$

To further understand the single exciton dynamics in this material, we performed time-resolved PL measurements of InAs core-only and InAs-CdSe core-shell NQD samples dissolved in hexane for a fixed InAs core diameter of 5.1 $\mathrm{nm}$ (excitation at $1.55 \mathrm{eV}$ ). Figure $2 \mathrm{e}$ shows that relaxation in the core-only material (monitored at $1.12 \mathrm{eV}$ ), which has an emission quantum yield of only $0.3 \%$, is biexponential and is dominated (75\% of the decay) by a subnanosecond relaxation time constant $(\sim 0.6 \mathrm{~ns}$ relaxation time measured with an instrument response function of $\sim 0.4 \mathrm{~ns}$ ) with a smaller amplitude $10.8 \mathrm{~ns}$ relaxation component. Deposition of a CdSe shell on this same InAs core results in a much higher emission quantum yield of $\sim 37 \%$ and markedly slower relaxation (monitored at $0.99 \mathrm{eV}$ ) that is single exponential with a time constant of $205 \mathrm{~ns}$. The multiexponential relaxation behavior of the core-only NQD sample indicates that the subnanosecond relaxation, which is also observed in single-exciton dynamics monitored with TA (Figure 2d), is likely due to carrier trapping rather than radiative relaxation, as has previously been reported for epitaxial ${ }^{35}$ and colloidal samples. ${ }^{34}$ Comparable results were obtained for other InAs core sizes. The very long excitedstate lifetime of the InAs-CdSe core-shell NQD material is notable given that the estimated radiative lifetime is on the order of $1 \mathrm{~ns}$ (following a calculation ${ }^{36}$ based upon bulk properties $^{37}$ ). The slow relaxation is also reminiscent of the long excited-state lifetime that is observed in studies of $\mathrm{PbSe}$ NQDs. ${ }^{36,38}$ Rationalization of the slow relaxation in $\mathrm{PbSe}$ NQDs has drawn upon consideration of the large dielectric screening correction of the optically active exciton radiative recombination rate. ${ }^{36}$ However, the same calculation applied to InAs NQDs does not provide for a reduced rate of radiative relaxation. Thus, we attribute the slow radiative relaxation in InAs NQDs to involvement of a low-lying, optically passive dark exciton state. ${ }^{39-41}$

The TA-based observation of a fast relaxation process at high pump intensity seen in Figure 2c,d does not conclusively demonstrate that the faster relaxation component is due to Auger recombination. In other NQD materials in which Auger recombination is well-established, a direct relationship between biexciton lifetime $\left(\tau_{\mathrm{XX}}\right)$ and NQD volume has been observed. ${ }^{1,23,24}$ Thus, to establish that the fast relaxation seen in TA dynamics at high pump intensity is due to Auger recombination, we measured pump-power dependent TA dynamics for a variety of InAs NQD sizes. However, because the utilized synthetic route to InAs NQDs provides undesirably broad NQD size/shape distributions ( $\sim 13 \%$ size dispersion is typical), which introduces uncertainty in the NQD dimensions, here we consider the relation of biexciton lifetime to the NQD confinement energy $E_{\mathrm{c}}=E_{\mathrm{g}}(\mathrm{NQD})-$ $E_{\mathrm{g}}$ (bulk). $E_{\mathrm{c}}$ is related to the NQD volume $V$ within the spherically symmetric confinement potential approximately as $V \propto E_{\mathrm{c}}^{-3 / 2}$.

In Figure 2f, we plot $\tau_{\mathrm{XX}}$ as a function of $E_{\mathrm{c}}^{-3 / 2}$ and observe a roughly linear relationship between these two quantities, which supports the interpretation of the fast relaxation observed at high pump intensity as Auger recombination of biexcitons. We also point out that the biexciton lifetimes of InAs core-only NQDs follow the same trend as that shown for InAs-CdSe core-shell NQDs. Additionally, $1 \mathrm{~S}$ exciton energies for InAs core-only and InAs-CdSe core-shell NQDs were related to the radius of InAs coreonly NQDs using the data from ref 20 to determine an 

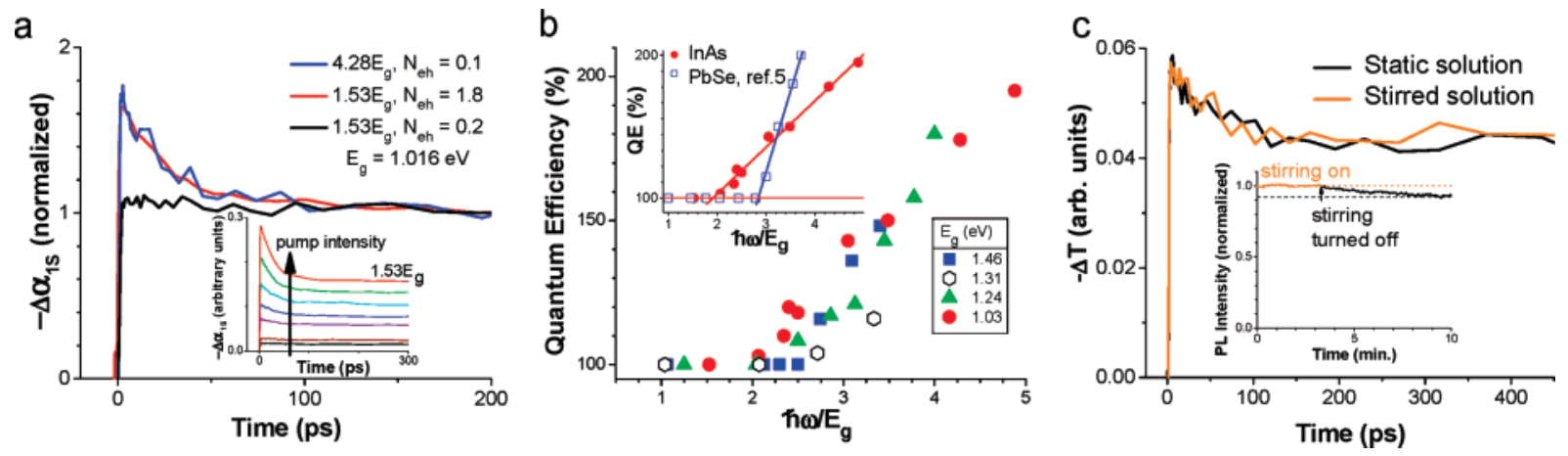

Figure 3. Carrier multiplication measurements in InAs-core and InAs-CdSe core-shell NQDs. (a) $1 \mathrm{~S}$ TA dynamics (normalized at long time, probe at $1.016 \mathrm{eV}$ ) of an InAs-CdSe core-shell NQD sample measured for three different excitation conditions: (1) Low photon energy $\left(1.53 E_{\mathrm{g}}\right)$ at low intensity $\left(N_{\text {eh }}=0.2\right.$, black line) to probe single exciton dynamics, $(2)$ high intensity $\left(N_{\text {eh }}=1.8\right.$, red line) to probe multiexciton dynamics, and finally (3) using high photon energy at low intensity $\left(4.28 E_{\mathrm{g}}, N_{\mathrm{eh}}=0.1\right.$, blue line) to probe multiexciton generation from a single photon. The dynamics indicate that the high photon energy excitation condition produces the same fast relaxation dynamics that are observed for multiexcitons produced at high pump fluences upon sequential absorption of multiple photons, which we have shown is due to Auger recombination of biexcitons. The bleach amplitude at early time $(t=1.5 \mathrm{ps})$ divided by the amplitude at long time ( $t=200 \mathrm{ps})$ (before and after Auger recombination, respectively) indicates the efficiency of CM for the high-photon energy excitation condition (blue line). Inset: development of multiexciton dynamics for increasing intensity using low-photon energy excitation (no CM). (b) CM quantum efficiency (number of excitons generated per absorbed photon) for several InAs-CdSe core-shell NQDs (solid blue squares, green triangles, red circles) and an InAs core-only NQD (open black hexagons) with indicated values of the material $E_{\mathrm{g}}$ (derived from the position of the $1 \mathrm{~S}$ absorption feature). Excitation photon energy is divided by the material energy gap $\left(\hbar \omega / E_{\mathrm{g}}\right)$. Inset: comparison of PbSe NQD CM efficiencies (blue open squares, from ref 5) with InAs (solid red circles) NQD CM efficiencies and shows a reduction of CM threshold for the III-V material to near $2 E_{\mathrm{g}}$. Pijpers et al. ${ }^{9}$ reported a CM efficiency of $160 \%$ at $2.74 E_{\mathrm{g}}$ in an InAs-CdSe-ZnSe core-shell-shell NQD sample, which is higher than the value reported here $(\sim 120 \%)$. (c) CM measurements (each collected in 5 min, data not normalized) with (orange line) and without (black line) solution stirring using $3.1 \mathrm{eV}$ excitation $\left(\mathrm{sample} \mathrm{OD}_{3.1 \mathrm{eV}}>4\right.$, excitation intensity of $6 \mu \mathrm{J} / \mathrm{cm}^{2}$ at $500 \mathrm{~Hz}, N_{\mathrm{eh}}<0.1$ ) and a $1.03 \mathrm{eV}$ probe. High optical density at the pump wavelength decreases the excitation volume and the corresponding residence time of NQDs in it. As a result, the probability of re-excitation of a given NQD on subsequent pump laser shots is reduced. Inset: NQD solution PL intensity is stable with solution stirring (orange line) and degrades over laboratory time when not stirred (black trace) to the level of the black dashed line. Measured CM efficiencies appear identical for both stirred and unstirred solutions.

effective NQD radius (this is an "effective" radius because the $1 \mathrm{~S}$ exciton energy shifts to lower energy upon growth of a CdSe shell). Biexciton lifetime plotted versus the NQD volume based on this effective NQD radius exhibits a linear relationship.

CM Onset and Efficiency. With an understanding of single- and multiexciton dynamics, we next perform measurements of $\mathrm{CM}$ efficiency. Figure 3a shows comparisons of TA dynamics (normalized at long time after Auger recombination has completed) that are collected using different excitation photon energies for an InAs-CdSe coreshell NQD. Development of Auger recombination dynamics using low-energy pump photons (no CM possible) with increasing pump intensity are shown as an inset. CM efficiencies as determined from the early time TA bleach maximum divided by the signal value at times after which only a single exciton per excited NQD remains (see Experimental Methods section) are shown in Figure $3 b$. Distinct from our previous $\mathrm{CM}$ studies on $\mathrm{PbSe}, \mathrm{PbS}$, and CdSe NQDs, in which we observe a fixed CM onset (quantified in terms of the number of energy gaps) above which CM efficiency grows linearly with a slope of $\sim 100 \%$ / $E_{\mathrm{g}}$ (see Figure $3 \mathrm{~b}$ inset), here we observe some NQD sizedependent variation in both the onset for $\mathrm{CM}$ and differential QE. Moreover, the CM onset appears to approach $2 E_{\mathrm{g}}$ as the $1 \mathrm{~S}$ exciton energy decreases (i.e., as the NQD size increases), which constitutes the apparent minimum energy required for generation of two excitons from a single photon.
Above this energy onset, we observe roughly linear growth of CM efficiency with a slope of $\sim 35 \% / E_{\mathrm{g}}$. CM is also observable in core-only InAs NQDs, although with a seemingly higher CM threshold and smaller differential QE than the (red-shifted) core-shell material. At present, we cannot comment definitively on the observed size- and passivation-dependent trends. We are currently analyzing these trends within different $\mathrm{CM}$ models $\mathrm{s}^{2,4,42-45}$ and expect that the observed variations may help to clarify the $\mathrm{CM}$ mechanism in NQDs.

During the course of our CM studies, we observed that PL intensity decreases as a function of laboratory time under illumination with high photon energies (see Figure $3 \mathrm{c}$ inset). We also noticed that degradation in the PL intensity does not occur if the sample is vigorously stirred. Photoinduced changes in the PL intensity observed for a "static" sample (no stirring) can result from a variety of processes (photochemical or photophysical). One possible effect is accumulation of long-lived charges in NQDs (NQD charging), which would reduce the PL intensity by introducing an additional nonradiative pathway for a photoexcited exciton via Auger recombination. Photoinduced charging can also lead to the development of a fast component in TA traces, which might be confused for the CM signal.

A distinct signature of long-lived charges in NQDs is a slow-relaxing bleaching of band-edge absorption, which persists on time scales longer than the period between two consecutive pump pulses ( $2 \mathrm{~ms}$ in these measurements). In 
our experiments, however, we do not detect any signatures of such bleaching (with 2\% accuracy) for either a "static" or a stirred sample, indicating that NQDs are not getting appreciably charged when excited with high-energy photons. We also performed CM measurements under "static" (where PL degradation is observed) and rapid stirring conditions (in which PL degradation is not observed). As can be seen in Figure $3 \mathrm{c}$, measured $\mathrm{CM}$ efficiencies are not affected by stirring and, hence, the effects leading to PL degradation apparently do not affect the CM process.

Exciton-Exciton Interaction Energy. We performed measurements to determine the Coulomb $\mathrm{X}-\mathrm{X}$ interaction strength in InAs NQDs because the energy required to produce a biexciton, and hence, the $\mathrm{CM}$ threshold, can be reduced by $\Delta_{\mathrm{Xx}}$. The values of $\Delta_{\mathrm{XX}}$ in larger epitaxial quantum dots of InAs have been reported to be $\sim 2-9 \mathrm{meV} .{ }^{46}$ Very recently, Pijpers et al. reported biexciton binding energies for InAs-CdSe-ZnSe core-shell-shell NQDs of $\sim 3-9 \mathrm{meV}$ (for $1 \mathrm{~S}$ exciton energy in the range of 0.85$1.05 \mathrm{eV}$ ) using a quasicontinuous-wave PL method. ${ }^{9}$ Here, we determine $\Delta_{\mathrm{Xx}}$ for an InAs-CdSe core-shell NQD sample using a spectrally resolved TA method. ${ }^{47,48}$ Spectrally resolved TA dynamics exhibit a photoinduced absorption feature on the low-energy side of the $1 \mathrm{~S}$ exciton transient bleach feature, which has a lifetime that is dictated by intraband relaxation of an excited exciton into the $1 \mathrm{~S}$ state. ${ }^{30}$ This photoinduced absorption feature originates from Coulomb-interaction-induced shift of the $1 \mathrm{~S}$ transition in the presence of a photoexcited exciton (the "biexciton shift" effect). ${ }^{47,48}$ The amplitude of this feature normalized by the amplitude of the $1 \mathrm{~S}$ exciton bleach directly relates to the magnitude of the shift (inset of Figure $4 \mathrm{~b}$ ) and, therefore, can be used to elucidate the $\mathrm{X}-\mathrm{X}$ interaction energy. ${ }^{47,48}$

Shown in Figure 4a is the TA spectrum measured for a pump delay that corresponds to the maximum transient signal amplitude (pump delay $<1 \mathrm{ps}$ ) for an $\mathrm{InAs}-\mathrm{CdSe}$ coreshell with a core diameter of $2.8 \mathrm{~nm}$ using low-intensity $1.77 \mathrm{eV}$ excitation. A photoinduced absorption that is redshifted from the $1 \mathrm{~S}$ bleach is clearly observable and transient dynamics measured at the amplitude maxima of the bleach $\left(B_{\mathrm{m}}\right)$ and induced absorption $\left(A_{\mathrm{m}}\right)$ (shown in Figure $4 \mathrm{~b}$ ) show that the decay of the induced absorption feature corresponds with the appearance of the $1 \mathrm{~S}$ bleach feature, typical of a biexciton shift. From analysis of the ratio $A_{\mathrm{m}} / B_{\mathrm{m}}$ and the line width of the $1 \mathrm{~S}$ transition, ${ }^{47,48}$ we obtain a value of $\Delta_{\mathrm{XX}}=$ $16 \mathrm{meV}$, which is somewhat larger than the values reported in ref 9 but might be expected given that the value reported here is for a smaller size InAs NQD core than in prior reports. Thus, the biexciton binding energy in InAs NQDs studied here represents $\sim 1 \%$ of the $1 \mathrm{~S}$ exciton energy and, therefore, will not produce a notable deviation of the $\mathrm{CM}$ onset below the expected threshold of $\sim 2 E_{\mathrm{g}}$. However, recently, we have demonstrated that by using appropriately engineered heteroNQDs, we can increase the $\mathrm{X}-\mathrm{X}$ interaction energy to more than $100 \mathrm{meV} \cdot{ }^{49,50}$ The purpose of this previous work was to obtain "giant" $\mathrm{X}-\mathrm{X}$ repulsion $\left(\Delta_{\mathrm{XX}}>0\right)$ as a means of achieving NQD lasing in the single-exciton regime. Currently, we are exploring strategies for obtaining strong $\mathrm{X}-\mathrm{X}$
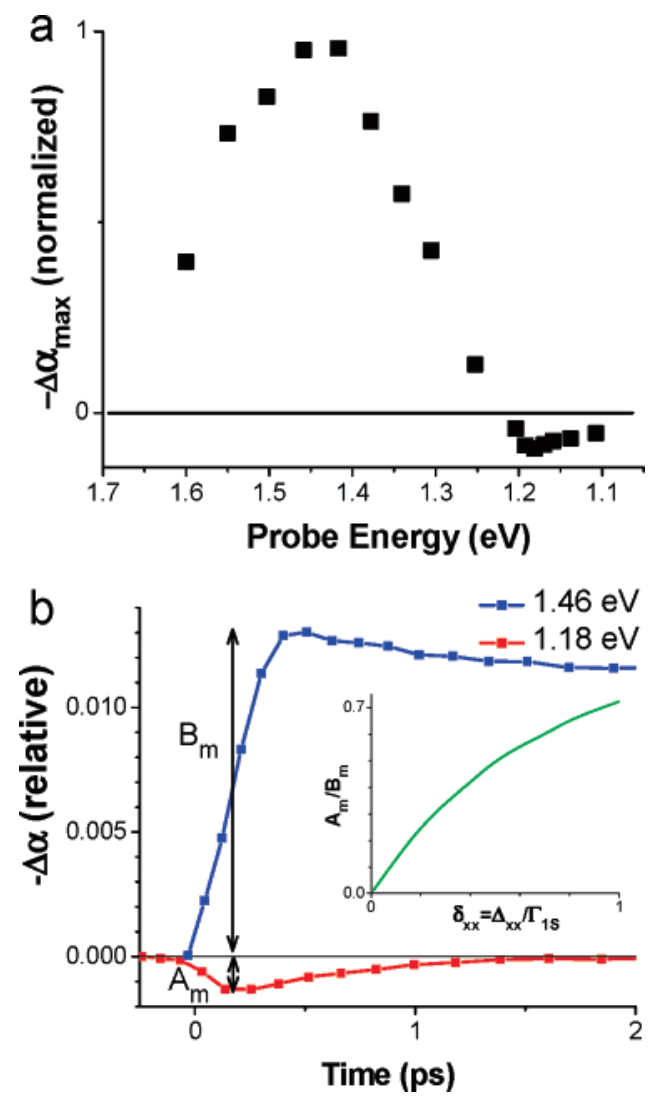

Figure 4. Transient-absorption-based measurements of the $X-X$ interaction energy. (a) TA spectrum collected for an InAs-CdSe core-shell NQD sample $\left(E_{\mathrm{g}}=1.55 \mathrm{eV}\right)$ using low intensity $\left(N_{\text {eh }}\right.$ $=0.1) 1.77 \mathrm{eV}$ excitation at temporal delays from the pump pulse that yield maximum bleach or photoinduced absorption amplitude (all pump to probe delays were between 0.15 and $0.6 \mathrm{ps}$ ). A small photoinduced absorption feature appears to the red of the strong 1S bleach feature. (b) TA dynamics measured at 1.46 and $1.18 \mathrm{eV}$ exhibit bleach and photoinduced absorption, respectively. The photoinduced absorption feature appears promptly and decays away in correlation with the appearance of the $1 \mathrm{~S}$ bleach feature. The ratio of transient amplitude maxima $\left(A_{\mathrm{m}}\right.$ and $\left.B_{\mathrm{m}}\right)$ combined with the line width of the $1 \mathrm{~S}$ transition $\left(\Gamma_{1 \mathrm{~S}}\right)$ are used to calculate the biexciton shift $\left(\Delta_{\mathrm{XX}}\right)$. The relation of these parameters is shown in the inset.

attraction $\left(\Delta_{\mathrm{XX}}<0\right)$ by manipulating both quantum and dielectric confinement effects in order to produce an appreciable reduction in the $\mathrm{CM}$ energy onset.

In conclusion, on the basis of the analysis of optical selection rules and energy conservation, we propose a criterion for the CM activation threshold in NQDs, which relates $\hbar \omega_{\mathrm{CM}}$ to the effective carrier masses. This criterion accurately predicts trends previously observed for $\mathrm{CM}$ onsets in semiconductor NQDs and indicates that the CM threshold can be reduced to the energy-conservation-defined minimum of $2 E_{\mathrm{g}}$ using materials with a significant difference between $m_{\mathrm{e}}$ and $m_{\mathrm{h}}$. By using InAs NQDs, for which $m_{\mathrm{e}} \ll m_{\mathrm{h}}$, we indeed observe $\mathrm{CM}$ thresholds that approach the fundamental $2 E_{\mathrm{g}}$ limit. We further discussed the possibility of obtaining the $\mathrm{CM}$ onset of less than $2 E_{\mathrm{g}}$ in the circumstance of strong Coulomb $\mathrm{X}-\mathrm{X}$ attraction, which would decrease the energy required to produce a biexciton compared to twice a singleexciton energy. The very low threshold reported here for 
$\mathrm{CM}$ in a III-V material indicates that these are promising candidates for application in CM-enhanced photovoltaics and photocatalytics. The observed differential QEs for these materials do not appear to be as large as for IV-VI NQDs; however, the low CM threshold is significant due to a larger flux of solar photons that are capable of producing multiexcitons.

Acknowledgment. This work was supported by the Chemical Sciences, Biosciences, and Geosciences Division of the Office of Basic Energy Sciences, Office of Science, U.S. Department of Energy and Los Alamos LDRD funds. J.M.P. is supported by an Intelligence Community postdoctoral research fellowship. V.I.K. acknowledges support by the Center for Integrated Nanotechnologies (CINT), a U.S. Department of Energy, Office of Basic Energy Sciences, nanoscale science research center operated jointly by Los Alamos and Sandia National Laboratories.

\section{References}

(1) Schaller, R. D.; Klimov, V. I. Phys. Rev. Lett. 2004, 92, 186601.

(2) Schaller, R. D.; Agranovich, V. M.; Klimov, V. I. Nat. Phys. 2005, $1,189$.

(3) Schaller, R. D.; Petruska, M. A.; Klimov, V. I. Appl. Phys. Lett. 2005, 87, 253102.

(4) Ellingson, R.; Beard, M. C.; Johnson, J. C.; Yu, P.; Micic, O. I.; Nozik, A. J.; Shabaev, A.; Efros, A. L. Nano Lett. 2005, 5, 865.

(5) Schaller, R. D.; Sykora, M.; Pietryga, J. M.; Klimov, V. I. Nano Lett. 2006, 6, 424.

(6) Schaller, R. D.; Klimov, V. I. Phys. Rev. Lett. 2006, 96, 097402.

(7) Schaller, R. D.; Sykora, M.; Jeong, S.; Klimov, V. I. J. Phys. Chem. B 2006, 110, 25332 .

(8) Murphy, J. E.; Beard, M. C.; Norman, A. G.; Ahrenkiel, S. P.; Johnson, J. C.; Yu, P. R. M.; O. I.; Ellingson, R. J.; Nozik, A. J. J. Am. Chem. Soc. 2006, 128, 3241.

(9) Pijpers, J. J. H.; Hendry, E.; Milder, M. T. W.; Fanciulli, R.; Savolainen, J.; Herek, J. L.; Vanmaekelbergh, D.; Ruhman, S.; Mocatta, D.; Oron, D.; Aharoni, A.; Banin, U.; Bonn, M. J. Phys. Chem. C 2007, 111, 4146.

(10) Klimov, V. I. Appl. Phys. Lett. 2006, 89, 123118.

(11) Hanna, M. C.; Nozik, A. J. J. Appl. Phys. 2006, 100, 074510.

(12) Koc, S. Czech. J. Phys. 1957, 7, 91

(13) Smith, A.; Dutton, D. J. Opt. Soc. Am. 1958, 48, 1007.

(14) Landsberg, P. Recombination in Semiconductors; Cambridge University Press: Cambridge, U.K., 1991.

(15) Klein, C. A. J. Appl. Phys. 1968, 39, 2029.

(16) Alig, R. C.; Bloom, S. Phys. Rev. Lett. 1975, 35, 1522.

(17) Efros, A. L.; Efros, A. L. Sov. Phys. Semicond. 1982, 16, 772

(18) Achermann, M.; Hollingsworth, J. A.; Klimov, V. I. Phys. Rev. B 2003, 68, 245302.
(19) Klimov, V. I. J. Phys. Chem. B 2006, 110, 16827.

(20) Guzelian, A. A.; Banin, U.; Kadavanich, A. V.; Peng, X.; Alivisatos, A. P. Appl. Phys. Lett. 1996, 69, 1432.

(21) Cao, Y.; Banin, U. J. Am. Chem. Soc. 2000, 122, 9692.

(22) Adachi, S. J. Appl. Phys. 1989, 66, 6030.

(23) Klimov, V. I. J. Phys. Chem. B 2000, 104, 6112.

(24) Klimov, V. I.; Mikhailovsky, A. A.; McBranch, D. W.; Leatherdale, C. A.; Bawendi, M. G. Science 2000, 287, 1011.

(25) Schaller, R. D.; Petruska, M. A.; Klimov, V. I. J. Phys. Chem. B 2003, 107, 13765 .

(26) Klimov, V. I.; McBranch, D. W.; Leatherdale, C. A.; Bawendi, M. G. Phys. Rev. B 1999, 60, 13740.

(27) Klimov, V. I.; Schwarz, C. J.; McBranch, D. W.; Leatherdale, C. A.; Bawendi, M. G. Phys. Rev. B 1999, 60, R2177.

(28) Klimov, V. I.; Mikhailovsky, A. A.; Xu, S.; Malko, A.; Hollingsworth, J. A.; Leatherdale, C. A.; Eisler, H.-J.; Bawendi, M. G. Science 2000, 290, 314.

(29) Malko, A. V.; Mikhailovsky, A. A.; Petruska, M. A.; Hollingsworth, J. A.; Klimov, V. I. J. Phys. Chem. B 2004, 108, 5250

(30) Klimov, V. I.; McBranch, D. W. Phys. Rev. Lett. 1998, 80, 4028.

(31) Blackburn, J. L.; Ellingson, R. J.; Micic, O. I.; Nozik, A. J. J. Phys. Chem. B 2003, 107, 102.

(32) Efros, A. L.; Kharchenko, V. A.; Rosen, M. Solid State Commun. 1995, 93, 281

(33) Wang, L.-W.; Califano, M.; Zunger, A.; Franceschetti, A. Phys. Rev. Lett. 2003, 91, 056404.

(34) Chen, G.; Rapaport, R.; Fuchs, D. T.; Lucas, L.; Aharoni, A.; Banin, U. Appl. Phys. Lett. 2005, 87, 251108.

(35) Karachinsky, L. Y.; Pellegrini, S.; Buller, G. S.; Shkolnik, A. S.; Gordeev, N. Y.; Evtikhiev, V. P.; Novikov, V. B. Appl. Phys. Lett. 2004, $84,7$.

(36) Wehrenberg, B. L.; Wang, C. J.; Guyot-Sionnest, P. J. Phys. Chem. $B$ 2002, 106, 10634.

(37) Hermann, C.; Weisbuch, C. Phys. Rev. B 1977, 15, 823.

(38) Du, H.; Chen, C.; Krishnan, R.; Krauss, T. D.; Harbold, J. M.; Wise, F. W.; Thomas, M. G.; Silcox, J. Nano Lett. 2002, 2, 1321.

(39) Efros, A. L.; Rosen, M.; Kuno, M.; Nirmal, M.; Norris, D. J.; Bawendi, M. G. Phys. Rev. B 1996, 54, 4843.

(40) Fu, H.; Wang, L.-W.; Zunger, A. Phys. Rev. B 1999, 59, 5568.

(41) Micic, O. I.; Cheong, H. M.; Fu, H.; Zunger, A.; Sprague, J. R.; Mascarenhas, A.; Nozik, A. J. J. Phys. Chem. B 1997, 101, 4904.

(42) Allan, G.; Delerue, C. Phys. Rev. B 2006, 73, 205423.

(43) Franceschetti, A.; An, J. M.; Zunger, A. Nano Lett. 2006, 6, 2191.

(44) Shabaev, A.; Efros, A. L.; Nozik, A. J. Nano Lett. 2006, 6, 2856.

(45) Rupasov, V. I.; Klimov, V. I. Phys. Rev. B 2007, 76, 125321.

(46) Sarkar, D.; van der Meulen, H. P.; Calleja, J. M.; Becker, J. M.; Haug, R. J.; Pierz, K. J. Appl. Phys. 2006, 100, 023109.

(47) Klimov, V.; Hunsche, S.; Kurz, H. Phys. Rev. B 1994, 50, 8110

(48) Klimov, V. I. Annu. Rev. Phys. Chem. 2007, 58, 635.

(49) Klimov, V. I.; Ivanov, S. A.; Nanda, J.; Achermann, M.; Bezel, I.; McGuire, J. A.; Piryatinski, A. Nature 2007, 447, 441.

(50) Piryatinski, A.; Ivanov, S. A.; Tretiak, S.; Klimov, V. I. Nano Lett. 2007, 7, 108

NL072046X 